\section{Safety and efficacy of HSCT for systemic sclerosis across clinical trials}

\author{
Lynette Keyes-Elstein, Erica Brittain, Ashley Pinckney, Ellen A. Goldmuntz and \\ Keith M. Sullivan (1)
}

Haematopoietic stem cell transplantation was superior to cyclophosphamide for the treatment of systemic sclerosis in three randomized clinical trials: ASSIST ${ }^{1}$, ASTIS $^{2}$ and SCOT ${ }^{3}$. Although all three trials infused autologous haematopoietic cells, they employed different preparative conditioning regimens, for which the relative safety and efficacy are unknown. We read with interest the News \& Views commentary by Burt and Farge (Systemic sclerosis: autologous HSCT is efficacious, but can we make it safer? Nat. Rev. Rheumatol. 14, 189-191 (2018)) $)^{4}$ comparing the results of these trials plus an uncontrolled retrospective study of the ASSIST regimen ${ }^{5}$. However, misapplied cross-study comparisons led to several faulty conclusions.

Burt and Farge asserted "the SCOT trial did not improve pulmonary function" ${ }^{\text {. How- }}$ ever, in a longitudinal analysis of forced vital capacity (FVC) trends, transplant was superior to cyclophosphamide ${ }^{6}$. The supplemental analysis they cited did not evaluate the treatment effect on FVC, as the event-free survival advantage of transplant is removed.

The statement that a myeloablative regimen "increases the risk of late-occurring cancer" requires clarification. The SCOT manuscript ${ }^{3}$ reported one case of breast cancer in the control arm, and two cases of myelodyplastic syndrome (MDS) and one of papillary (not medullary) thyroid cancer in the transplant group by 6 years. Because MDS is rare, it is reasonable to deduce that MDSrisk increases with myeloablation relative to control, but evidence does not support an increased risk of cancer in general. Each arm had one non-MDS cancer, and the time-at-risk was shorter in the cyclophosphamide group owing to greater deaths and early withdrawals. Importantly, none of these manuscripts ${ }^{1-3,5}$ provides a cumulative incidence of cancers over time accounting for duration of follow-up and numbers at-risk. Because the number of cases is very small, precise comparisons of rates are not possible. Some 'late-occurring' cancers might also have been missed, as the ASTIS manuscript ${ }^{2}$ would have excluded any non-fatal cancers occurring after 2 years, and in the ASSIST report only 17 individuals were followed beyond 3 years. Hence, the relative risk of cancers for myeloablative and non-myeloablative regimens cannot be reliably evaluated.

The statement that "Transplant-related mortality for the SCOT trial was equivalent to that for the ASSIST trial and lower than for the ASTIS trial" simplifies a nuanced issue. For ASTIS ${ }^{2}$ and ASSIST $^{5}$, transplant-related deaths occurred during the first year; however, no transplant deaths occurred during this period in $\mathrm{SCOT}^{3}$. Transplant-related deaths in SCOT occurred later, at 16 and 68 months, and were secondary to MDS. Also, Burt and Farge $^{4}$ claimed that the incidence of "major (grade 4) transplant-related adverse events" (AEs) was higher for SCOT than ASTIS, but cited event rates that are not comparable. ASTIS $^{2}$ reported grade 4 events accrued over 2 years; SCOT $^{3}$ reported grade 4 and 5 events accrued over 6 years. Furthermore, reporting rules for haematologic AEs may have differed, as red blood cell and platelet transfusions are indicative of severe haematologic AEs and were reported as such in SCOT but not in ASTIS $^{2}$. Other cross-trial differences between the reports also warrant careful consideration.

When comparing studies with different treatments, end points, statistical methodology, follow-up duration and study populations, careful interpretation of results is essential to ensure appropriate insights into the relative efficacy and safety of treatments ${ }^{7}$.

Lynette Keyes-Elstein ${ }^{1}$, Erica Brittain ${ }^{2}$, Ashley Pinckney ${ }^{1}$, Ellen A. Goldmuntz ${ }^{2}$ and Keith M. Sullivan (D) $^{3 \times}$

${ }^{1}$ Rho Federal Systems, Durham, NC, USA.

${ }^{2}$ National Institute of Allergy and Infectious Diseases, Bethesda, MD, USA

${ }^{3}$ Duke University Medical Center, Durham, NC, USA.

凶e-mail:keith.sullivan@duke.edu https://doi.org/10.1038/s41584-020-0493-2

1. Burt, R. K. et al. Autologous non-myeloablative haemopoietic stem-cell transplantation compared with pulse cyclophosphamide once per month for systemic sclerosis (ASSIST): an open-label, randomised phase 2 trial. Lancet 378, 498-506 (2011).

2. van Laar, J. M. et al. Autologous hematopoietic stem cell transplantation versus intravenous pulse cyclophosphamide in diffuse cutaneous systemic sclerosis: a randomized clinical trial. JAMA 311, 2490-2498 (2014).

3. Sullivan, K. M. et al. Myeloablative autologous stem-cell transplantation for severe scleroderma. N. Engl. J. Med. 378, 35-47 (2018).

4. Burt, R. K. \& Farge, D. Systemic sclerosis: autologous HSCT is efficacious, but can we make it safer? Nat. Rev. Rheumatol. 14, 189-191 (2018).

5. Burt, R. K. et al. Cardiac involvement and treatmentrelated mortality after non-myeloablative haemopoietic stem-cell transplantation with unselected autologous peripheral blood for patients with systemic sclerosis: a retrospective analysis. Lancet 381, 1116-1124 (2013).

6. Keyes-Elstein, L. et al. Longitudinal trends in clinical disease features after myeloablative autologous stem-cell transplantation or cyclophosphamide in severe scleroderma [abstract]. Arthritis Rheum. 70 (suppl. 10), 902 (2018).

7. Sullivan, K. M. \& Keyes-Elstein, L. Cross trial comparisons in clinical practice reviews: proceed with caution. Nat. Rev. Rheumatol. https://doi.org/ 10.1038/s41584-020-0492-3 (2020).

\section{Competing interests}

The authors declare no competing interests. 